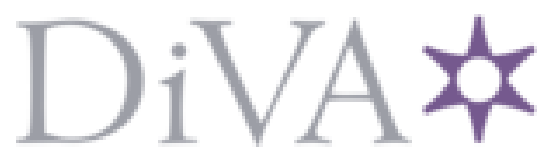

http://www.diva-portal.org

\title{
Postprint
}

This is the accepted version of a paper presented at 2oth European Conference on Circuit Theory and Design (ECCTD 2011), Linköping, Sweden, August 29-31, 2011.

Citation for the original published paper:

Nilsson, E., Svensson, C. (2011)

Envelope Detector Sensitivity and Blocking Characteristics

In: 2011 2oth European Conference on Circuit Theory and Design (ECCTD), 6043845

(pp. 773-776). Piscataway, NJ: IEEE Press

https://doi.org/10.1109/ECCTD.2011.6043845

N.B. When citing this work, cite the original published paper.

Permanent link to this version:

http://urn.kb.se/resolve?urn=urn:nbn:se:hh:diva-16342 


\section{Envelope Detector Sensitivity and Blocking Characteristics}

\author{
Emil Nilsson \\ CERES and MPE-lab \\ Halmstad University \\ Halmstad, SE-301 18, Sweden \\ Email: emil.nilsson@hh.se
}

\author{
Christer Svensson \\ ISY \\ Linköping University \\ Linköping, SE-581 83, Sweden \\ Email: christer@isy.liu.se
}

\begin{abstract}
This paper presents analytical expressions for the sensitivity of a low power envelope detector driven by a weak RF signal in the presence of a blocking signal. The envelope detector has been proposed for low power Wake-Up radios in applications such as RFID and wireless sensor systems. The theoretical results are verified with simulations of a modern short channel MOS transistor in a commonly used circuit topology. A discussion around a tutorial example of a radio frontend, consisting of an LNA and a detector, is presented. It is shown that the sensitivity of a low power envelope detector can reach $-62 \mathrm{dBm}$ with a low power LNA and in presence of a $\mathrm{CW}$ blocker.
\end{abstract}

\section{INTRODUCTION}

For a long time development of radio technology has focused on complicated high performance modulation schemes like QAM rather than simple OOK (on-off keying). However, recent developments in ultra low power radio, with applications such as RFID and wireless sensor systems have showed the need for simple wake up radios [1][2][3]. A wake up radio is intended to be in constant operation, listening on the air interface for a wake up signal. Once receiving this signal the wake up radio may start more power demanding and sophisticated functional blocks. The requirements for the wake up radio thus are, ultra low power consumption, robust and reliable functionality, and a reasonable sensitivity. OOK modulation is a simple and robust non-coherent modulation, not requiring an always running local oscillator.

The input voltage amplitude for the wake up radio detector is far below the traditional levels of a few hundred millivolts $(k T / q)$ normally preferred to drive nonlinear components efficiently. The reason for these low levels is the wish to minimize RF amplification since this normally is a power consuming part of the radio. Internal noise in the detector together with noise from the preceding RF stages sets the lower limit of the detectable amplitude level. In addition to this noise, external interfering sources may swamp the wake up signal, causing interference and increased noise due to noise folding.

A theory is needed for the performance of the system in these conditions. In this paper we develop such a theory together with analytical expressions for detector sensitivity. All expressions are based on the exponential transfer function of the subthreshold biased transistor. The validity of this simplified model is verified by simulations of a modern short

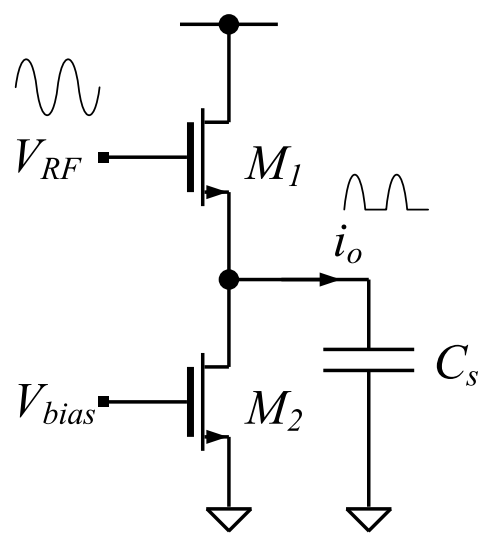

Fig. 1. Envelope detector schematic.

channel MOS transistor in a commonly used circuit topology. Further the use of the derived expressions is exemplified by analysis of a hypothetical detector in combination with an LNA. The circuit in Fig. 1 is a common implementation of an envelope detector [4], transformed from a bipolar to a CMOS circuit. M1 is biased in subthreshold, where it displays a maximum nonlinear transfer function. Transistor M2 acts as a constant bias current source, keeping M1 gate bias voltage $V_{g s 0}$ constant. The detector output voltage is found over capacitor $C_{s}$ which is charged by the output current $i_{o}$. This capacitor also provides the RF signal grounding path. The detection of the RF-signal is performed by the nonlinear transfer function from voltage to current in M1. Detector efficiency depends on how abrupt the nonlinear transfer function is. MOS transistors in subthreshold region of operation display an exponential nonlinearity at room temperature.

The paper is organized as follows; II) Theory for the envelope detector. III) Verification of theory with simulations. IV) Discussion. V) Conclusion.

\section{THEORY FOR THE ENVELOPE DETECTOR}

Assume that we use an NMOS transistor in subthreshold for detection. Then the $I_{d}-V_{g s}$ characteristic is

$$
I_{d}=I_{d 0} e^{V_{g s} / V_{0}}
$$


where $V_{0}=n k T / q$ with $1<n<2$. The gate to source voltage may be expanded to,

$$
V_{g s}=V_{g}+V_{g s 0},
$$

where $V_{g}$ is the small signal voltage, represented by $V_{R F}$ in Fig. 1, and $V_{g s 0}$ is the bias voltage. For small amplitudes of $V_{g}$ (meaning small deviations from $V_{g s 0}$ ) we may, assuming any DC bias being included in $I_{d 0}$, expand (1) as

$$
I_{d}=I_{d 0}+\frac{d I_{d}}{d V_{g}} V_{g}+\frac{d^{2} I_{d}}{d V_{g}^{2}} \frac{V_{g}^{2}}{2} .
$$

Where the second derivative is

$$
\frac{d^{2} I_{d}}{d V_{g}^{2}}=\frac{I_{d 0}}{V_{0}^{2}} .
$$

With an applied unmodulated RF signal we get $V_{g}=V_{s} \cos (\omega t)$ and the quadratic term above can be expanded as $\cos ^{2}(\omega t)=$ $(1+\cos 2 \omega t) / 2$. We may now calculate the wanted incremental component in the output signal current $i_{o}$ that is dependent on amplitude $V_{s}$ as:

$$
i_{s}=I_{d 0} \frac{V_{s}^{2}}{4 V_{0}^{2}}
$$

The output noise current contributors may be divided into three parts. Noise from the channel of the detector transistor, baseband noise being transferred without any frequency conversion, and noise at radio frequencies converted to baseband. All these contributors have spectral components in the baseband making them worth study. Noise self mixing components will also have spectral components in baseband, but the level from this contribution will be so low that it is negligible.

The channel noise will not vary with the input signal levels since it is a function of the bias current being kept constant in our circuit. The output noise current from the NMOS transistor can be expressed as [5]:

$$
i_{n}^{2}=4 \gamma k T g_{m} B
$$

where $g_{m}=d I_{d} / d V_{g}=I_{d 0} / V_{0}, \gamma \approx 1$, and $B$ is the noise bandwidth. From this we can estimate SNR to

$$
S N R=\frac{i_{s}^{2}}{i_{n}^{2}}=\frac{g_{m} V_{s}^{4}}{64 k T V_{0}^{2} B} .
$$

We have here defined SNR from an incremental current $i_{s}$, assuming that our main interest is OOK modulation. We can then estimate the minimum $V_{s}$ for a particular SNR giving us the envelope detector sensitivity,

$$
V_{\text {smin }}=\sqrt[4]{\frac{64 k T V_{0}^{2} B}{g_{m}} S N R .}
$$

For a reasonably low power consumption $(<1 \mu \mathrm{A})$, we may have $g_{m}=10 \mu \mathrm{A} / \mathrm{V}$. Further assuming $B=100 \mathrm{kHz}$ and $\mathrm{SNR}=10$, we arrive to a sensitivity of $2.2 \mathrm{mV}$ (peak, corresponding to $-43 \mathrm{dBm}$ at $50 \Omega$ ).

The second noise component, baseband noise leakage, can be kept very low by shorting the input to ground at the baseband frequencies.

\section{A. The detector sensitivity in presence of a blocking signal}

The third noise component is RF noise being down converted to baseband. This RF noise is converted to baseband by mixing with the carrier or with an in band blocker. We will limit our analysis to first order, i.e. mixing with the first harmonic of the blocker signal only.

In the noise calculation we do not consider the effects of signal driven cyclic variation of the transconductance, since the transconductance is kept constant by the constant bias current source. If we add a blocker of amplitude $V_{b}$, we will have to modify the input signal component to $V_{g}=V_{s} \cos (\omega t)+$ $V_{b} \cos \left(\omega_{b} t\right)$. However, the blocker is not necessarily small, so we may rewrite (1) as

$$
\begin{aligned}
I_{d}= & I_{d 0} e^{\frac{V_{s}}{V_{0}} \cos \omega t} e^{\frac{V_{b}}{V_{0}} \cos \omega_{b} t}= \\
& I_{d 0}\left(1+\frac{V_{s}}{V_{0}} \cos \omega t+\frac{V_{s}^{2}}{2 V_{0}^{2}} \cos ^{2} \omega t\right) e^{\frac{V_{b}}{V_{0}} \cos \omega_{b} t}(9)
\end{aligned}
$$

The second exponent can be expanded as a Fourier series [4]:

$$
e^{\frac{V_{b}}{V_{0}} \cos \omega_{b} t}=\mathcal{I}_{0}\left(\frac{V_{b}}{V_{0}}\right)+2 \mathcal{I}_{1}\left(\frac{V_{b}}{V_{0}}\right) \cos \omega_{b} t+\cdots
$$

Where $\mathcal{I}_{0}$ and $\mathcal{I}_{1}$ are modified Bessel functions of the first kind. This means that the term we are interested in, the incremental current term proportional to $V_{s}^{2}$, can be written:

$$
i_{s}=I_{d 0} \mathcal{I}_{0}\left(\frac{V_{b}}{V_{0}}\right) \frac{V_{s}^{2}}{4 V_{0}^{2}}
$$

From (10) it is clear that a large blocking signal will have an influence on the biasing conditions of the transistor. As a matter of fact an increased current due to a blocker will increase the detector sensitivity, but also risking the transistor to be pulled out of the subthreshold region. In our actual circuit, see Fig. 1, the DC-current is kept constant through the bias current generator to $I_{\text {bias }}$. Therefore we have

$$
I_{d 0} \mathcal{I}_{0}\left(\frac{V_{b}}{V_{0}}\right)=I_{\text {bias }},
$$

so we can expect the sensitivity (5), and the internal transistor channel noise current (6), to be unaffected by the blocker power. However, the output DC voltage from the circuit will change with blocker power.

In the presence of a blocker signal, the blocker will act as a local oscillator and convert some of the RF noise to baseband. Strictly speaking also the signal may act as local oscillator, but by assuming the signal to be weak we can neglect this contribution. Replacing $V_{s}$ in (9) with input noise amplitude $V_{n i}$ at frequency $\omega_{n}$ gives,

$$
i_{n b}=I_{d 0}\left(1+\frac{V_{n i}}{V_{0}} \cos \omega_{n} t\right) e^{\frac{V_{b}}{V_{0}} \cos \omega_{b} t},
$$

where we use only the first order noise term. Again utilizing the Fourier expansion of the exponential function, the resulting noise in baseband can be expressed as

$$
i_{n b}^{2}=2 I_{d 0}^{2} \frac{V_{n i}^{2}}{V_{0}^{2}} \mathcal{I}_{1}^{2}\left(\frac{V_{b}}{V_{0}}\right)
$$




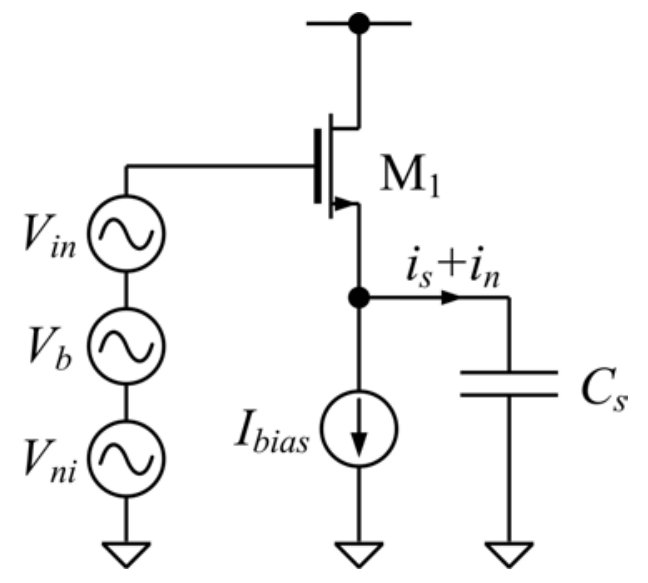

Fig. 2. Simulated circuit with back gate connected to source.

where we have considered both sidebands of the RF noise. Combining (14) with (12) gives the following expression for our circuit

$$
i_{n b}^{2}=2 I_{\text {bias }}^{2} \frac{\mathcal{I}_{1}^{2}\left(V_{b} / V_{0}\right)}{\mathcal{I}_{0}^{2}\left(V_{b} / V_{0}\right)} \frac{V_{n i}^{2}}{V_{0}^{2}}
$$

\section{B. Comparison of blocker-induced noise and transistor noise}

In order to understand the relevance of this expression, let us compare this blocker-induced noise with the transistor noise in our circuit. For this purpose, we describe the input RF noise to the transistor as its equivalent noise resistor, $R_{n i}$, defined by

$$
V_{n i}^{2}=4 k T R_{n i} B
$$

We then use (15) and (6) and set $i_{n b}=i_{n}$ and arriving to

$$
R_{n i}=\frac{\gamma}{2 g_{m}} \frac{\mathcal{I}_{0}^{2}\left(V_{b} / V_{0}\right)}{\mathcal{I}_{1}^{2}\left(V_{b} / V_{0}\right)}
$$

where we also used $I_{b i a s} / V_{0}=g_{m}$. With typical values of $g_{m}$ for a low power detector, say $10 \mu \mathrm{A} / \mathrm{V}$, we note that $\gamma / 2 g_{m}$ is of the order of $50 \mathrm{k} \Omega$. As $\mathcal{I}_{0}(x) / \mathcal{I}_{1}(x)$ approaches 1 for large $x, R_{n i}$ is never lower than $50 \mathrm{k} \Omega$, indicating that even for very large blockers (or signals), we can allow quite large input RF noise without affecting the total noise current or SNR. For $V_{b} / V_{0}=1, R_{n i}=112 \mathrm{k} \Omega$, showing that lower blocker power will further increase the RF input noise level not affecting SNR.

\section{VERIFICATION OF THEORY WITH SIMULATIONS}

The above theory was tested against simulations in EDA software (ADS [6]). The circuit used is depicted in Fig. 2, where M2 in Fig. 1 is replaced with an ideal current generator for simplicity. The voltage $V_{i n}$ drives the transistor with an $\mathrm{AM}$ modulated $\mathrm{RF}$ signal, a superimposed blocker signal generator $V_{b}$ and an external RF noise source, $V_{n i}$. The AM modulated signal is defined as

$$
V_{i n}=V_{m} \frac{1+\sin \omega_{m} t}{2} \cos \omega t
$$

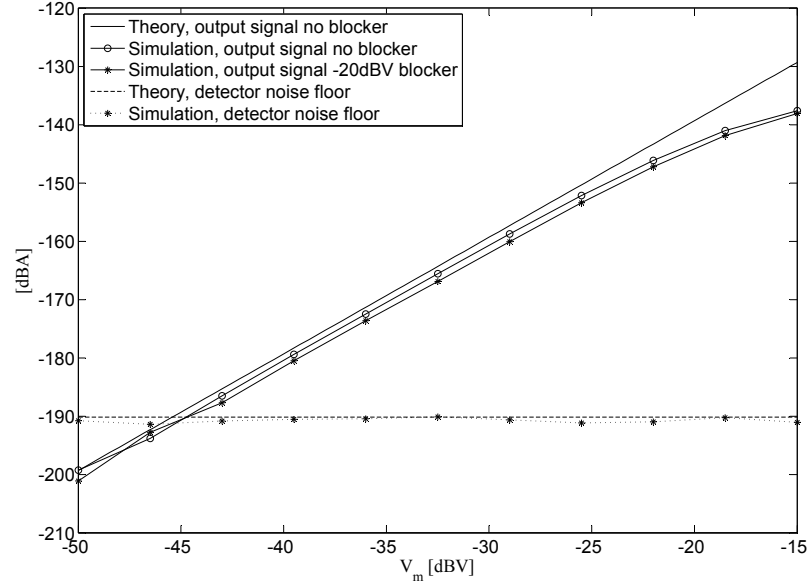

Fig. 3. Output signal current $i_{s m}$ and output noise current $i_{n}$ for envelope detector as a function of modulated RF amplitude $V_{m}$.

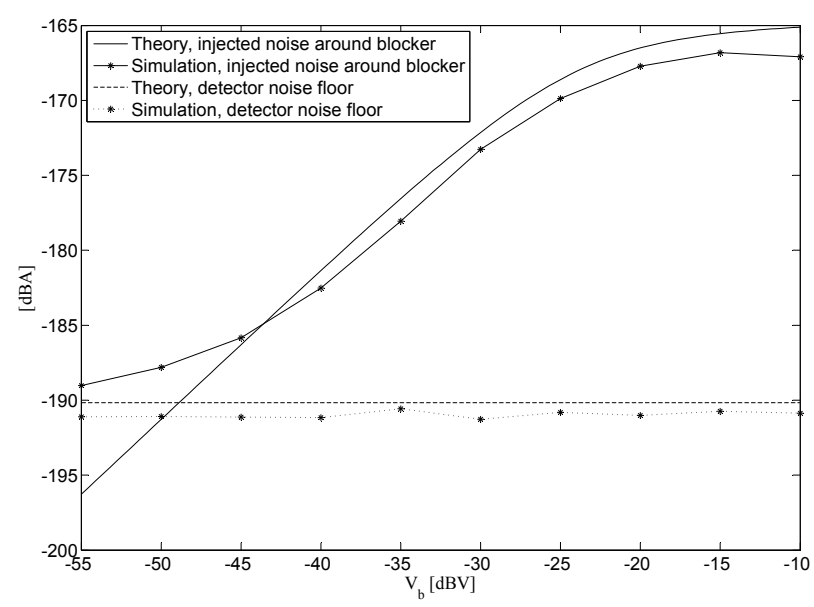

Fig. 4. Output noise current $i_{n b}$ for the envelope detector as a function of blocker amplitude $V_{b}$ at a corresponding injected external noise amplitude of $V_{n i}=-57 \mathrm{dBV}$ in (15). The injected noise is distributed in a band $\pm 10 \mathrm{MHz}$ around the blocker with a density of $1 \mu \mathrm{V} / \sqrt{\mathrm{Hz}}$. The modulated RF signal amplitude is $V_{m}=-54 \mathrm{dBV}$.

where $\omega_{m}$ is the modulation angular frequency. The parameter $V_{m}$ is defined as the maximum RF amplitude during the modulation cycle. The external RF noise source has its spectrum around the blocker frequency and is zero around baseband. The output signal amplitude at angular frequency $\omega_{m}$ is half the incremental signal shift stated in (5), thus

$$
i_{s m}=\frac{1}{8} \frac{I_{d 0}}{V_{0}^{2}} V_{m}^{2} .
$$

The simulation results are plotted together with the values predicted by theory in Fig. 3 and Fig. 4.

Fig. 3 shows a good agreement between theory ((5) and (19)) and simulations except for the largest signal amplitudes, where the deviation from $V_{g s 0}$ is not small and the approximation using the expansion in (4) does not hold. Also Fig. 4 shows a good agreement between theory and simulations. For low blocker amplitudes noise flattens out to a constant level. One possible explanation is that the detector noise is 
dominated by noise self mixing rather than noise folding at these lower blocker levels. Another conceivable explanation may be the modulated RF signal starts to dominate over the blocker signal as driver of noise folding. Preliminary simulations and measurements indicate noise self mixing as the most probable mechanism.

\section{Discussion}

The investigated envelope detector can be used either on its own or in combination with an LNA as a wake-up receiver RF frontend. As the basic sensitivity is not affected by an inband blocker, we anticipate that a receiver using this envelope detector can operate also in the presence of an inband blocker considerably larger than the signal itself. The only effects of a blocker is 1) its amplitude modulation will affect the receiver if that spectrum falls within the baseband spectrum used by the receiver, and 2) it may convert a large input RF noise to noise in the receiver baseband. In addition, intermodulation products between the blocker and the signal may affect the receiver, depending of their respective spectra. Standing alone, the envelope detector sensitivity is about $2.2 \mathrm{mV}$ peak at $100 \mathrm{kHz}$ bandwidth. With a relatively high impedance antenna (antenna with matching network) of, say, $300 \Omega$, this corresponds to a sensitivity of $-50.9 \mathrm{dBm}$. For a standalone detector the input RF noise will not be large enough to disturb the SNR in the presence of a blocker. If a larger sensitivity is required, we need to add an LNA in front of the detector. With an LNA with power gain $G_{L N A}$ and noise figure $F_{L N A}$ we improve sensitivity by $G_{L N A}$. In the same time the input noise voltage squared to the detector will be $k T B R_{r e f} G_{L N A} F_{L N A}$, where $R_{\text {ref }}$ is the reference impedance at the LNA output. The RF noise voltage at the input to the envelope detector is thus equivalent to a noise resistance of $R_{r e f} G_{L N A} F_{L N A}$, which thus should be smaller than $R_{n i}$ from (17) in order not to affect SNR by a blocker. Therefore if we limit $G_{L N A} F_{L N A}$ to about 150 (assuming $R_{\text {ref }}=300 \Omega$ ), blocker converted noise will be of no importance. This may for example correspond to $F_{L N A}=10$ (10 dB; high in order to save LNA power) and $G_{L N A}=15(12 \mathrm{~dB})$, improving the sensitivity to $-62.9 \mathrm{dBm}$.

\section{CONCLUSION}

We have analyzed the sensitivity of an envelope detector, with and without the presence of an inband blocker. It was shown that a stand alone detector can achieve a sensitivity of about $-50 \mathrm{dBm}$ at a power consumption of less than $1 \mu \mathrm{W}$, unaffected by an inband blocking CW signal or signal with low AM-modulation depth inside the Wake-Up radio modulation bandwidth. We further showed that the sensitivity can be increased to about $-62 \mathrm{dBm}$ with an uncritical LNA, again unaffected by a blocker.

\section{ACKNOWLEDGMENT}

The authors would like to thank Peter Linnér at the department of Microelectronics and Nanoscience at Chalmers University of Technology for fruitful discussions. This work has been performed within the ELLIIT strategic research initiative funded by the Swedish government.

\section{REFERENCES}

[1] D. C. Daly and A. P. Chandrakasan, "An energy-efficient OOK transceiver for wireless sensor networks," IEEE J. Solid-State Circuits, vol. 42, pp. 1003-1011, May 2007.

[2] N. M. Pletcher, S. Gambini, and J. M. Rabaey, "A $52 \mu \mathrm{W}$ wake-up receiver with $-72 \mathrm{dBm}$ sensitivity using an uncertain-IF architechture," IEEE $J$. Solid-State Circuits, vol. 44, pp. 269-280, Jan. 2009.

[3] X. Huang, S. Rampu, X. Wang, G. Dolmans, and H. de Groot, "A $2.4 \mathrm{GHz} / 915 \mathrm{MHz} 51 \mu \mathrm{W}$ wake-up receiver with offset and noise suppression," in IEEE International Solid-State Circuits Conference 2010, Feb. 9,2010 , pp. 222-224.

[4] R. Meyer, "Low-power monolithic RF peak detector analysis," IEEE $J$. Solid-State Circuits, vol. 30, pp. 65-67, Jan. 1995.

[5] B. Razavi, Design of Analog CMOS Integrated Circuits. Boston: McGraw-Hill, 2001.

[6] Agilent. (2010) Advanced design system (ADS). [Online]. Available: www.agilent.com/find/ads 